\title{
Quantifizierung des pflanzlichen Strandanwurfs an der westdeutschen Ostseeküste
}

\author{
H. Grave $\mp$ \& H. Möller \\ Institut für Meereskunde an der Universität; \\ Düsternbrooker Weg 20, D-2300 Kiel, Federal Republic of Germany
}

\begin{abstract}
Quantification of dead marine plant biomass along the Baltic Sea coast of Western Germany. Following evaluation of 634 aerial photographs, the dead marine plant biomass on the western Baltic Sea shores between Flensburg and Neustadt (Federal Republic of Germany) was estimated to be $300 \mathrm{t}$ dry weight in August 1977 . On the average, a belt of 0,9 m width was covered by dead marine plant material. Main constituents were Fucus vesiculosus L. and Zostera marina L. The impact of the hydrographical conditions on the quantity of plants washed ashore is discussed.
\end{abstract}

\section{EINLEITUNG}

In der fast tidenfreien Kieler Bucht haben die küstenparallelen Oberflächenströmungen durch Substrattransport den größten Einfluß auf die makrobenthische Vegetation (Schwenke, 1971). Zusammensetzung und Menge des pflanzlichen Strandanwurfs werden durch die Produktion benthischer Algen und Seegräser sowie durch die hydrographischen und meteorologischen Gegebenheiten bestimmt. Langfristige Strömungsmessungen aus dem Flachwasserbereich $(0-10 \mathrm{~m})$ der Kieler Bucht liegen bisher nicht vori auch die Produktion des Makrophytobenthos ist bisher nur wenig bekannt. Hoffmann (1952) schätzte den Fucus-Bestand bis hinab zur $6 \mathrm{~m}$ Tiefenlinie im untersuchten Gebiet auf $4 \times 10^{4} \mathrm{t}$ oder $1,8 \mathrm{~kg} / \mathrm{m}^{2} \mathrm{NaBgewicht}$.

Mit Hilfe von Schneisenprofilen und Dauerbeobachtungen untersuchte Black (1978) die Strukturdynamik der Algen unter besonderer Berücksichtigung des $0-18 \mathrm{~m}$ Bereichs. Er errechnete für die gesamte Kieler Bucht eine Algenbiomasse zwischen $15 \times 10^{4}$ und $52 \times 10^{4}$ t. Eigenen Beobachtungen zufolge wird im Flachwasser der Kieler Bucht neben Algen ein bedeutender Teil makrobenthischer Biomasse durch Zostera marina produziert. Nach Sand-Jensen (1975) produzieren Seegräser der nahegelegenen Vellerup Vig etwa $1 \mathrm{~kg}$ Trockengewicht pro $\mathrm{m}^{2}$ und Jahr. Ein Teil dieser pflanzlichen Produktion wird an den Strand gespült, wo er in Fäulnisprozessen zersetzt, verweht, oder an Badestränden maschinell entfernt wird. Uber die Dauer von Austrocknungs- und Fäulnisprozessen berichtet Schramm (1968).

Die vorliegende Untersuchung stellt eine erste Abschätzung der Menge des im Spätsommer vorgefundenen Strandanwurfs dar. 


\section{METHODE}

Im August 1977 wurden entlang der 320 km langen Küstenlinie zwischen Flensburg und Neustadt 634 Kleinbild-Luftaufnahmen $(24 \times 36 \mathrm{~mm})$ angefertigt. Einzelpositionen sind in Abbildung 1 aufgeführt. Die Flughöhe betrug $330 \mathrm{~m}$, die Länge des pro Bild aufgenommenen Küstensaumes ca. $170 \mathrm{~m}$ (Hochformat) oder $270 \mathrm{~m}$ (Querformat). Die durch den Aufnahmewinkel von ca. $30^{\circ}$ verzerrten Fotos wurden durch das Auflegen eines Rasters entzerrt, das entsprechend der photographischen Verzerrung von Hafenanlagen mit bekannten Vermessungen angefertigt wurde. Durch das Raster ließen sich die Fotos in $5 \times 5 \mathrm{~m}$ große Felder einteilen. Die mit Anwurf bedeckten Felder wurden ausgezählt und die mittlere Breite des Anwurfs errechnet. In Abbildung 1 ist die durchschnittliche Anwurfbreite für Punkte mit jeweils $500 \mathrm{~m}$ Abstand in Balkenform dargestellt. Es wurde stets das gleitende Mittel über 3 Punkte errechnet. Fehlende Werte zwischen zwei Aufnahmen wurden graphisch ermittelt.

\section{ERGEBNISSE UND DISKUSSION}

Die mittlere Breite des Anwurfs entlang des gesamten Küstensaumes betrug ca. $0,9 \mathrm{~m}$. Bei einer Küstenlänge von $320 \mathrm{~km}$ entspricht dies einer Gesamtfläche von $0,3 \mathrm{~km}^{2}$. Nach der Auswertung von je zwei Stichproben von $0,25 \mathrm{~m}^{2}$ an jeweils 10 verschiedenen Küstenorten ergab sich ein Trockengewicht des von Sand- und Muschelschalen gereinigten pflanzlichen Strandanwurfs von $1,0 \mathrm{~kg} / \mathrm{m}^{2}\left(0,5-1,4 \mathrm{~kg} / \mathrm{m}^{2}\right)$. Die Gesamtmenge der angespülten Pflanzen kann demnach auf $300 \mathrm{t}$ geschätzt werden.

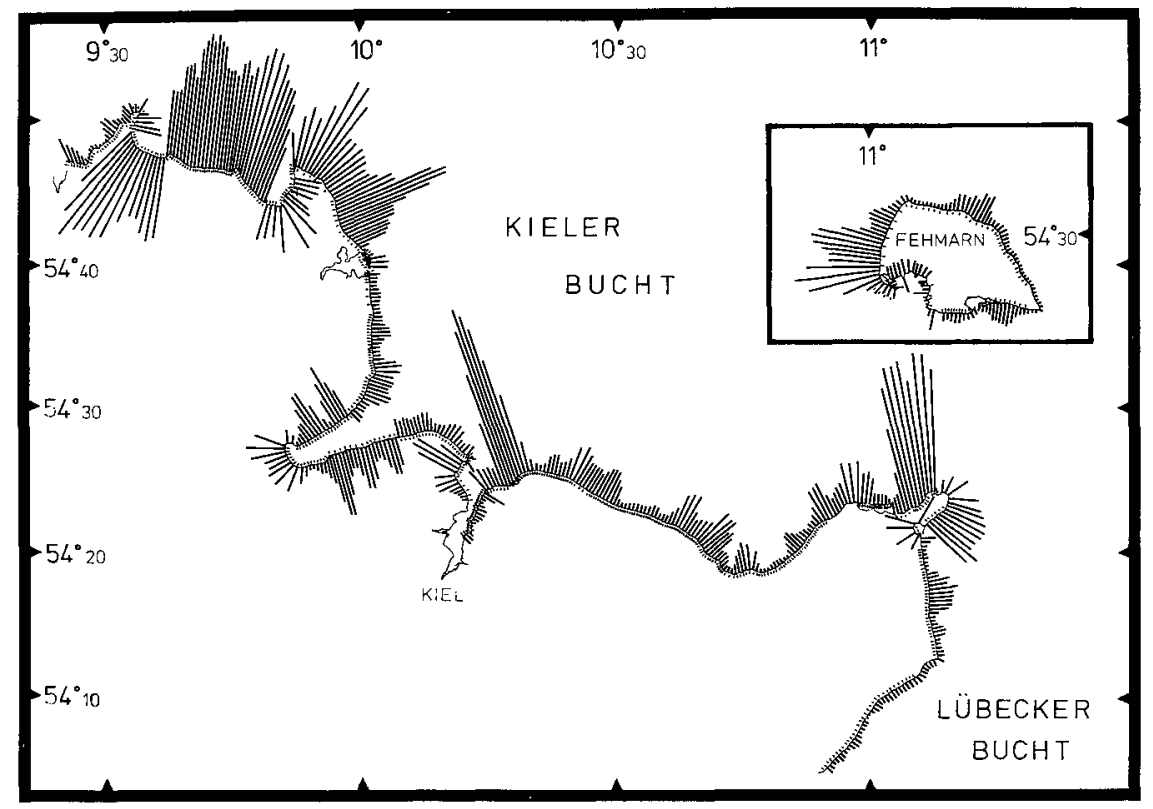

Abb. 1. Bodenbedeckung durch pflanzlichen Strandanwurf im August 1977. $1 \mathrm{~cm}$ Balkenlänge entspricht einer Anwurfbreite von $1 \mathrm{~m}$. Die Positionen einzelner Aufnahmen sind durch Rauten gekennzeichnet 
Es bestanden erhebliche regionale Unterschiede in der Menge des Strandanwurfs. Relativ wenig Anwurf war entlang der offenen Küsten zu finden, die Winden aus 0-180 ausgesetzt sind. Nur die östlichen Ufer von Halbinseln (Birk, Großenbrode) stellten Ausnahmen dar. In intensiv genutzten Erholungsgebieten (Lübecker Bucht) wird der Anwurf maschinell beseitigt.

Relativ dichter Anwurf trat in geschützten Förden auf, ebenso an Küstenstrichen, die westlichen Winden ausgesetzt sind. Dem Bottsand und der Halbinsel Großenbrode kommt dabei offenbar ein Falleneffekt zu.

Nach unseren Beobachtungen finden sich über das gesamte Jahr gesehen die größten Pflanzenmengen im Frühwinter am Strand, nachdem die abgestorbenen Zostera-Blätter angespült wurden. Sturm und Küsteneis reißen im Spätwinter dann zeitweise zusätzlich erhebliche Mengen Blasentang vom Untergrund los und führen somit zu vermehrtem Anwurf.

Über den Anteil des Anwurfs an der gesamten Produktion des Phytobenthos sind zur Zeit nur grobe Schätzungen möglich. Unter der Annahme, daß der Fucus-Bestand sich im Laufe von 3 Jahren regeneriert (Hoffmann, 1952), werden bei einem Gesamtbestand von $4 \times 10^{4}$ t Naßgewicht jährlich $1,3 \times 10^{4} \mathrm{t}$ produziert. Dieser Wert entspricht einem Trockengewicht von $2 \times 10^{3}$ t. Der Strandanwurf im August 1977 setzte sich zu drei Vierteln aus Fucus vesiculosus $\left(2 \times 10^{2}\right.$ t) und zu einem Viertel aus anderen Algenarten und Seegras zusammen. Somit endeten $10 \%$ der geschätzten jährlichen Fucus-Produktion im sommerlichen Strandanwurf.

\section{ZITIERTE LITERATUR}

Black, H., 1978: Vegetationsdynamische Untersuchungen an epilithischen Algengemeinschaften im Sublitoral der westlichen Ostsee unter besonderer Berücksichtigung des Problems der produktionsbiologischen Bestandsabschätzung. Diss. Univ. Kiel, 134 S.

Hoffmann, C., 1952: Über das Vorkommen und die Menge industriell verwertbarer Algen an der Ostküste Schleswig-Holsteins. - Kieler Meeresforsch. 9, 5-14.

Sand-Jensen, K., 1975: Biomass, net production and growth dynamics in an eelgrass (Zostera marina L.) population in Vellerup Vig, Denmark. - Ophelia 14, 185-201.

Schramm, W., 1968: Ökologisch-physiologische Untersuchungen zur Austrocknungs- und Temperaturresistenz an Fucus vesiculosus L. der westlichen Ostsee. - Int. Revue ges. Hydrobiol. 53, $463-510$.

Schwenke, H., 1971: Meeresbotanische Untersuchungen in der westlichen Ostsee als Beitrag zu einer marinen Vegetationskunde. - Int. Revue ges. Hydrobiol. 54, 35-94. 